infarct size was observed when WT-MI hearts were compared to their PMCA1 ${ }^{\mathrm{Ht}}$ counterparts 1 week post-surgery. Interestingly, whilst both WT and PMCA1 $1^{\mathrm{Ht}}$ MI-treated mice showed a significant deterioration in cardiac function 1 week post-surgery there were differences seen in cardiac structure between the two groups. For example, echocardiography revealed that the WT-MI hearts were significantly more hypertrophic when compared to their PMCA1 ${ }^{\mathrm{Ht}}$ counterparts shown by a significant increase in the LV diameters alongside a significant difference in the normalised heart weight to tibia length ratios. ECG revealed significantly longer QT intervals and considerably more extra-systolic events among the WT-MI mice. Whilst histological analysis of cardiomyocyte size showed a significantly exacerbated hypertrophic response in the remote regions of WT-MI mice compared to PMCA1 ${ }^{\mathrm{Ht}}-\mathrm{MI}$ mice.

Conclusion Heterozygous deletion of PMCA1 might serve a protective role in the heart following both moderate and severe MI. The protective mechanism most likely develops in the early post-operative phase, as an MI of similar extent is associated with higher mortality rates in WT mice. Future work will aim to elucidate the mechanism producing this phenotype mainly focusing on the potential role that PMCA1 might serve in the process.

\section{HERITABILITY AND FAMILY-BASED GWAS ANALYSES TO DISCOVER NOVEL LIPIDOMIC BIOMARKERS OF CARDIOVASCULAR DISEASE}

${ }^{1}$ Kathryn McGurk*, ${ }^{2}$ Anna Nicolaou, ${ }^{1}$ Bernard Keavney. ${ }^{1}$ Division of Cardiovascular Sciences, University of Manchester; ${ }^{2}$ Division of Pharmacy and Optometry, University of Manchester

\subsection{6/heartinl-2017-311726.140}

Introduction Lipids have key roles in CVD but heritability studies focus on few species bar lipoproteins. Lipids play key roles in cell signalling, immunity, inflammation, vasodilation and cell death. Although not encoded, their activities are tightly linked to DNA-encoded entities (e.g. enzymes and other proteins) and those with a strong genetic influence (high heritability) may identify novel pathways in CVD. Purpose: Analysing eicosanoid, endocannabinoid and sphingolipid profiles in 250 British Caucasian families with GWAS data will identify particularly heritable lipid biomarkers for the discovery of causative genetic variation of CVD.

Methods An array of 79 eicosanoids and related species, 33 endocannabinoids and congeners, 63 ceramides and related species, from 204 plasma samples (31 families of $1+$ individuals with hypertension) were extracted and analysed by liquid chromatography coupled to tandem mass spectrometry (LCMS/MS). Quality control was accessed and estimates of heritability of each lipid species was estimated using QTDT software. The concentration of each lipid species were assessed for normal distribution, outliers, and adjusted for age and sex. The resulting lipid concentrations will be analysed using FaSTLMM software for associations with 557,124 SNPs.

Results 19 species of eicosanoids were identified (mean concentrations $19 \mathrm{pg} / \mathrm{ml}-7600 \mathrm{pg} / \mathrm{ml}$ ); the species at highest abundance in plasma (HODEs) are derivatives of the omega- 6 fatty acid linoleic acid. 8 species passed quality control assessments and 3 species were estimated to be significantly heritable (9-OxoODE 24\%, 12-HETE 44\%, 12(13)-EPOME 78\%). 15 congeners of endocannabinoids were identified (mean concentrations $20 \mathrm{pg} / \mathrm{ml}-4000 \mathrm{pg} / \mathrm{ml}$ ); the species at highest abundance in plasma are glycerol derivatives. 9 species passed quality control assessments and all were estimated to be significantly heritable $(27 \%-73 \%) \%) .57$ species of ceramides were identified (mean concentrations $0.01 \mathrm{pmol} / \mathrm{ml}-190 \mathrm{pmol} / \mathrm{ml}$ ); the species at highest abundance in plasma are NS ceramides involved in apoptosis. 36 species passed quality control assessments and all species were estimated to be significantly heritable (10\%-63\%)\%).

Conclusions We demonstrate for the first time estimates of heritability for an array of bioactive lipids. Although found at concentrations million times lower than cholesterol, many of the heritability estimates are similar to plasma genetic biomarkers of CVD (ACE h2>45\%). The association between heritable biomarkers, cardiovascular phenotypes and risk will be determined. Particularly heritable species and those with highly significant hits at GWAS will be profiled in the extended cohort (1400 samples, 250 families) and replication will take place in the UK biobank, or other. A subset of the most heritable species and those with hits at GWAS resulting from the full lipidomic profile of 3 lipidomic classes will be used to identify causal metabolic pathways, novel diagnostics and drug targets for CVD intervention.

\section{INTRAVITAL IMAGING OF LEUKOCYTE, PLATELET AND STEM CELL TRAFFICKING IN VIVO IN THE CARDIAC MICROCIRCULATION FOLLOWING MYOCARDIAL ISCHAEMIA-REPERFUSION INJURY}

Dean Kavanagh*, Adam Lokman, Neena Kalia. University of BirminghamStem cells, Reperfusion injury, Imaging

10.1136/heartjnl-2017-311726.141

Background The clinical success of stem cell (SC) therapy for myocardial infarction is compromised by poor cardiac homing following systemic delivery. As such therapy may depend on beneficial paracrine effects, it is a further hindrance that little is known about the inflammatory response dynamics within myocardial microcirculation in vivo.

Methods 3D-printed stabilisers were bonded to the beating heart of anaesthetised (ketamine/xylazine) mice to enable confocal intravital imaging of ventricular microcirculation. PE-antiGr-1 and APC-anti-CD41 antibodies were injected to label neutrophils and platelets respectively with FITC-BSA enabling blood flow visualisation. In some mice, haematopoietic SCs (HSCs; HPC-7s) were introduced intra-arterially. IR injury was induced by 45 min (reversible) ligation of the LAD artery.

Results Neutrophil adhesion and platelet accumulation were both significantly $(p<0.001)$ and rapidly increased in injured microvessels with platelet accumulation increasing with time. No difference in number or velocity of free-flowing neutrophils was observed. A significant $(p<0.05)$ decrease in functional capillary density was also observed in injured hearts. Although HSC adhesion was not significantly enhanced following injury, a time-dependent increase in adhesion was observed in sham and injured hearts. No significant change in number or velocity of free-flowing HSCs was observed following injury. Interestingly, despite reduced capillary perfusion, approximately 10-20 HSCs were observed trafficking though the heart at each time point throughout reperfusion.

Discussion Intravital microscopy has allowed successful visualisation of the microvascular inflammatory response and HSC homing events in the beating mouse heart post-reperfusion. 